\title{
Atomic scattering from an adsorbed monolayer solid with a helium beam that penetrates to the substrate
}

\author{
Hansen, Flemming Yssing; Bruch, L.W.; Dammann, Bernd
}

Published in:

Journal of Chemical Physics

Link to article, DOI:

$10.1063 / 1.4794742$

Publication date:

2013

Document Version

Publisher's PDF, also known as Version of record

Link back to DTU Orbit

Citation (APA):

Hansen, F. Y., Bruch, L. W., \& Dammann, B. (2013). Atomic scattering from an adsorbed monolayer solid with a helium beam that penetrates to the substrate. Journal of Chemical Physics, 138(10), 104705.

https://doi.org/10.1063/1.4794742

\section{General rights}

Copyright and moral rights for the publications made accessible in the public portal are retained by the authors and/or other copyright owners and it is a condition of accessing publications that users recognise and abide by the legal requirements associated with these rights.

- Users may download and print one copy of any publication from the public portal for the purpose of private study or research.

- You may not further distribute the material or use it for any profit-making activity or commercial gain

- You may freely distribute the URL identifying the publication in the public portal 


\section{AIP $\begin{gathered}\text { molowanat } \\ \text { chemical Physics }\end{gathered}$}

Atomic scattering from an adsorbed monolayer solid with a helium beam that penetrates to the substrate

F. Y. Hansen, L. W. Bruch, and B. Dammann

Citation: J. Chem. Phys. 138, 104705 (2013); doi: 10.1063/1.4794742

View online: http://dx.doi.org/10.1063/1.4794742

View Table of Contents: http://jcp.aip.org/resource/1/JCPSA6/v138/i10

Published by the American Institute of Physics.

Additional information on J. Chem. Phys.

Journal Homepage: http://jcp.aip.org/

Journal Information: http://jcp.aip.org/about/about_the_journal

Top downloads: http://jcp.aip.org/features/most_downloaded

Information for Authors: http://jcp.aip.org/authors

\section{ADVERTISEMENT}
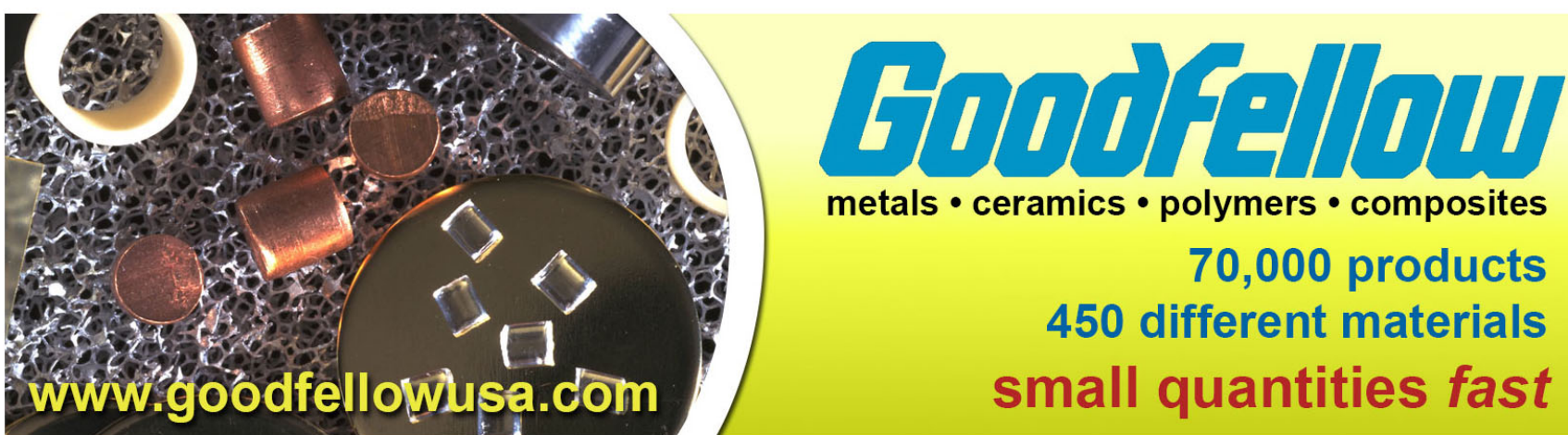
metals • ceramics $\bullet$ polymers $\bullet$ composites 70,000 products 450 different materials small quantities fast 


\title{
Atomic scattering from an adsorbed monolayer solid with a helium beam that penetrates to the substrate
}

\author{
F. Y. Hansen, ${ }^{1, \text { a) }}$ L. W. Bruch, ${ }^{2}$ and B. Dammann ${ }^{3}$ \\ ${ }^{1}$ Department of Chemistry, Technical University of Denmark, IK-207-DTU, DK-2800 Lyngby, Denmark \\ ${ }^{2}$ Department of Physics, University of Wisconsin-Madison, 1150 University Avenue, Madison, \\ Wisconsin 53706, USA \\ ${ }^{3}$ Department of Informatics and Mathematical Modeling, Technical University of Denmark, 321-DTU, \\ DK 2800 Lyngby, Denmark
}

(Received 18 December 2012; accepted 22 February 2013; published online 14 March 2013)

\begin{abstract}
Diffraction and one-phonon inelastic scattering of a thermal energy helium atomic beam are evaluated in the situation that the target monolayer lattice is so dilated that the atomic beam penetrates to the interlayer region between the monolayer and the substrate. The scattering is simulated by propagating a wavepacket and including the effect of a feedback of the inelastic wave onto the diffracted wave, which represents a coherent re-absorption of the created phonons. Parameters are chosen to be representative of an observed $p(1 \times 1)$ commensurate monolayer solid of $\mathrm{H}_{2} / \mathrm{NaCl}(001)$ and a conjectured $p(1 \times 1)$ commensurate monolayer solid of $\mathrm{H}_{2} / \mathrm{KCl}(001)$. For the latter, there are cases where part of the incident beam is trapped in the interlayer region for times exceeding $50 \mathrm{ps}$, depending on the spacing between the monolayer and the substrate and on the angle of incidence. The feedback effect is large for cases of strong transient trapping. () 2013 American Institute of Physics. [http://dx.doi.org/10.1063/1.4794742]
\end{abstract}

\section{INTRODUCTION}

Inelastic helium atom scattering experiments have determined the energies of phonons in physically adsorbed monolayer solids. ${ }^{1,2}$ The theory is relatively complex because the processes that make a thermal energy He beam an exquisitely surface sensitive probe require treating the scattering as a strong coupling event. ${ }^{3-6}$ For example, the simply commensurate monolayer solid $p(1 \times 1) \mathrm{H}_{2} / \mathrm{NaCl}(001)$ is such a corrugated target that some experimental diffraction peaks are more intense than the specular peak ${ }^{7}$ and the theory ${ }^{6}$ shows this too. Further, that monolayer solid is so dilated that there is only a low potential energy barrier for the He to penetrate to the interlayer region that is between the plane of the monolayer molecule centers and the plane of the centers of surface ions of the substrate and there may be paths with no barrier for a more dilated conjectured commensurate monolayer solid $p(1 \times 1) \mathrm{H}_{2} / \mathrm{KCl}(001)$ that has not yet been observed.

We anticipated that there would be strong transient trapping in the interlayer region for $\mathrm{He}$ scattering from $p(1 \times 1) \mathrm{H}_{2} / \mathrm{KCl}(001)$ and found in trial calculations that the wavepacket scattering methodology extended stably to such conditions. However, the strong coupling effects are enhanced for the wave function in the interlayer region. Therefore, we have generalized the one-phonon scattering theory to include a feedback of the inelastic wave function onto the elastic (diffracted) wave function, in which the created phonon is coherently annihilated. This provides a self-consistent (SC) description of the scattering process in the zero and one phonon space and maintains conservation of total probability.

\footnotetext{
a) Author to whom correspondence should be addressed. Electronic mail: flemming@kemi.dtu.dk.
}

We have re-visited the $p(1 \times 1) \mathrm{H}_{2} / \mathrm{NaCl}(001)$ system, because including the barrier penetration effect satisfactorily removes a norm loss artifact in the calculations of Ref. 6, denoted as Paper III here. There are only minor changes from the previously calculated inelastic scattering distributions. For this system there are good models ${ }^{8-10}$ for the He$\mathrm{H}_{2}$ and $\mathrm{He}-\mathrm{NaCl}$ interactions and experimental data ${ }^{7}$ for the phonon spectrum. In contrast, the components of the interaction model for the $\mathrm{H}_{2} / \mathrm{KCl}(001)$ are less well-determined and the calculations there are more exploratory in character. As yet, there are no inelastic helium atom scattering experiments that show effects of large penetration to the interlayer region between monolayer and substrate. This is in marked contrast to low energy electron diffraction experiments, where interference between reflections at the monolayer and at the substrate is analyzed to determine the adsorption-site geometry. ${ }^{11}$

Our previous work (Papers I-III) ${ }^{4-6}$ is in the context of the time-dependent evolution of a scattering wave packet and starts from the one-phonon scattering approximation of Choi and Poe. ${ }^{12}$ The time-dependent theory exhibits the dynamic interplay of the elastic and inelastic processes. There also is a large body of work $^{3}$ on helium atom scattering from physically adsorbed monolayers that uses a time-independent coupled-channel formulation. From its beginning, ${ }^{13}$ approximate solutions were constructed which conserved total probability (unitarity) and that was implemented with averaged approximations for the Debye-Waller factor. According to a recent example of such work, ${ }^{14}$ our self-consistent onephonon calculations may over-estimate the effect of onephonon losses; this is likely to become a large effect for multiphonon scattering ${ }^{3}$ which we do not treat here. Another related effect is the phonon-assisted resonances ${ }^{15,16}$ in which one-phonon processes enhance selective adsorption 
resonances in the diffracted wave. Although the cases in our model calculation are not at the resonant condition, there are long transient trapping times that suggest a proximity to such effects. The role of thermal excitation of the monolayer and dynamics of the substrate is not yet incorporated in our work. We believe that this may be done more easily in the time-dependent theory than in the time-independent formulation but that the two approaches have an important complementarity. As calculations are extended to higher energies where multiphonon processes contribute more, semi-classical approximations ${ }^{17}$ will play a larger role.

The organization of this paper is as follows: Sec. II summarizes the theoretical components of this work, the potential models, and the scattering theory. Section III presents the results of the calculations. Section IV contains the conclusions. The Appendix summarizes a ray tracing (geometrical optics) approximation for the penetration of $\mathrm{He}$ to the interlayer region.

\section{THEORY}

\section{A. Self-consistent one-phonon approximation}

The Hamiltonian for scattering of an atom by the monolayer solid is

$$
H=K_{\text {kin }}+H_{\text {ph }}+V_{c} .
$$

$K_{\text {kin }}$ is the kinetic energy operator of the incident $(\mathrm{He})$ atom of mass $m_{a} . H_{\mathrm{ph}}$ is the Hamiltonian of the monolayer solid treated in the harmonic approximation and is expressed in phonon operators by

$$
H_{\mathrm{ph}}=\sum_{\mathbf{Q}, \lambda} \hbar \omega(\mathbf{Q}, \lambda) a^{+}(\mathbf{Q}, \lambda) a(\mathbf{Q}, \lambda),
$$

with a sum over wavevectors $\mathbf{Q}$ in the first Brillouin zone and polarization branches $\lambda$. The ground state of $H_{\mathrm{ph}}$ is denoted $\Phi_{0}$, with energy zero, i.e., zero-point energies are omitted. $V_{c}$ denotes the sum of the atom-monolayer and atom-substrate potential energies. It consists of a static part $V_{s}$ with all monolayer and substrate atoms at their equilibrium positions and a dynamic part $V_{d}$ arising from the vibrational displacements of the monolayer atoms. Dynamic coupling of the monolayer and substrate is neglected and $V_{d}$ is approximated by $V_{1}$, its expansion to first order in monolayer atom displacements and phonon operators:

$$
V_{d} \simeq V_{1}=\sum_{\mathbf{Q}, \lambda} \tilde{V}_{\mathbf{Q}, \lambda} a^{+}(\mathbf{Q}, \lambda)+\tilde{V}_{\mathbf{Q}, \lambda}^{*} a(\mathbf{Q}, \lambda) .
$$

The time-dependent Schrödinger equation then is

$$
\imath \hbar \frac{\partial \Psi}{\partial t}=\left(H_{0}+H_{\mathrm{ph}}+V_{1}\right) \Psi,
$$

with $H_{0}=K_{\text {kin }}+V_{s}$. Assuming a solution of the form $\Psi=\Psi_{0} \Phi_{0}+\sum_{\mathbf{Q}, \lambda} \Psi_{1}(\mathbf{Q}, \lambda) a^{+}(\mathbf{Q}, \lambda) \Phi_{0}$ and projecting Eq. (4) onto the zero-phonon $\left(\Phi_{0}\right)$ and one-phonon $\left(a^{+}(\mathbf{Q}, \lambda) \Phi_{0}\right)$ spaces gives coupled equations for the scattered-atom wave functions $\Psi_{0}$ and $\Psi_{1}$

$$
\imath \hbar \frac{\partial \Psi_{0}}{\partial t}=H_{0} \Psi_{0}+\sum_{\mathbf{Q}, \lambda} \tilde{V}_{\mathbf{Q}, \lambda}^{*} \Psi_{1}(\mathbf{Q}, \lambda)
$$

and

$$
\iota \hbar \frac{\partial \Psi_{1}(\mathbf{Q}, \lambda)}{\partial t}=\left[H_{0}+\hbar \omega(\mathbf{Q}, \lambda)\right] \Psi_{1}(\mathbf{Q}, \lambda)+\tilde{V}_{\mathbf{Q}, \lambda} \Psi_{0} .
$$

There is an Eq. (6) for each $(\mathbf{Q}, \lambda)$ in the sum in Eq. (5). Multiphonon processes are neglected and the phonon states are those of the unperturbed monolayer. The total norm is conserved for this set of dynamical equations:

$$
\begin{aligned}
\left(\Psi_{0}+\Psi_{1}, \Psi_{0}+\Psi_{1}\right) & =\left(\Psi_{0}, \Psi_{0}\right)+\left(\Psi_{1}, \Psi_{1}\right) \\
& =\int\left[\left|\Psi_{0}\right|^{2}+\sum_{\mathbf{Q}, \lambda}\left|\Psi_{1}(\mathbf{Q}, \lambda)\right|^{2}\right] d x d y d z=1 .
\end{aligned}
$$

In a scattering event with initial condition $\Psi_{1}=0$ and the monolayer initially in the state $\Phi_{0}$, the last term in Eq. (6) usually acts as a source term. There are time periods where its action is more complex and the norm of $\Psi_{1}$ does not increase monotonically with time. The $\tilde{V}_{\mathbf{Q}, \lambda}^{*}$ term in Eq. (5) represents the feedback of the inelastic scattering onto the elastic (diffraction) scattering and leads to a reduction in the norm of $\Psi_{0}$ from the initial value of one. This self-consistency represents one of the processes in the Debye-Waller factor for diffraction intensities. ${ }^{3}$ We denote solutions of Eqs. (5) and (6) by SC and those in which the $\tilde{V}_{\mathbf{Q}, \lambda}^{*}$ term is omitted from Eq. (5), as in Paper III, by NSC (non-self-consistent).

The lateral, $\mathbf{r}=(x, y)$, periodicities of the potential energies $V_{s}(\mathbf{r}, z)$ and $\tilde{V}_{\mathbf{Q}, \lambda}(\mathbf{r}, z)$ are expanded in the Steele series $^{19}$ using the reciprocal lattice vectors $\mathbf{g}$ of the monolayer. The corresponding expansions of the zero-phonon and one-phonon atom wave functions $\Psi_{0}$ and $\Psi_{1}$ are, with $\mathbf{K}$ the initial parallel wavevector of the atom,

$$
\Psi_{0}=\sum_{\mathbf{g}} \psi_{\mathbf{g}}(z, t) \exp [l(\mathbf{g}+\mathbf{K}) \cdot \mathbf{r}]
$$

and

$$
\Psi_{1}(\mathbf{Q}, \lambda)=\sum_{\mathbf{g}} \exp [\imath(\mathbf{K}-\mathbf{Q}+\mathbf{g}) \cdot \mathbf{r}] S_{\mathbf{Q}, \lambda}(\mathbf{g}, z, t) .
$$

\section{B. Implementation: Helium atom scattering}

\section{Interaction models}

The interaction of the $\mathrm{He}$ atom with an $\mathrm{H}_{2}$ molecule is taken to be the TSM model ( $\mathrm{Tao}^{8}$ and Spelsberg-Meyer ${ }^{9}$ ) without zero-point averaging, as given in Paper III. However, in evaluating the coefficients in the Steele series, a cutoff procedure is adopted for small separations because the numerical integration of Eqs. (5) and (6) becomes unstable for very large potential energies. A smooth cutoff is used at separations $r$ less than a value $r_{s}$ :

$$
V(r)=V_{\mathrm{TSM}}(r) \times \exp \left(-\gamma_{2}\left[r-r_{s}\right]^{2}\right), \quad r \leq r_{s} .
$$

With the choices $r_{s}=1.5 \AA$ and $\gamma_{2}=0.421 \AA^{-2}$, the maximum of $\mathrm{V}(\mathrm{r})$ is limited to $13.1 \mathrm{eV}$ and the wavepacket propagates stably even when a significant amount penetrates to the interlayer region between the monolayer and the substrate. Since the He beam energies considered are in the range 
8-26 meV, this cutoff on $V(r)$ is unlikely to give spurious effects in the scattering calculation. The He-monolayer potential is the sum of $V_{\mathrm{TSM}}$ or $V$ over a square lattice of $\mathrm{H}_{2}$ molecules with nearest-neighbor spacing $L_{n n}=3.96 \AA$ [ 4.44 $\AA]$ for the $\mathrm{NaCl}(001)[\mathrm{KCl}(001)]$ case.

The interaction of $\mathrm{He}$ with $\mathrm{NaCl}(001)$ was modeled by Vargas and Mochán. ${ }^{10}$ In Paper III, the effective surface plane of the $\mathrm{NaCl}$ is taken to be $D Z L=1.2 \AA$ below the $\mathrm{H}_{2}$ monolayer; the actual distance from the $\mathrm{H}_{2}$ monolayer to the $\mathrm{NaCl}(001)$ is calculated ${ }^{18}$ to be $2.6 \AA$. A similar construction for $\mathrm{He}-\mathrm{KCl}(001)$ is not available, but the selective adsorption energy levels are known ${ }^{7}$ to be very similar to those for $\mathrm{He}-$ $\mathrm{NaCl}(001)$. As an estimate of the $\mathrm{He}-\mathrm{KCl}(001)$ potential we adopt the Vargas-Mochán model and use three values for $D Z L$ $(1.2,2.8$, and $4.0 \AA)$. The larger values lead to transient configurations with large interlayer components in both $\Psi_{0}$ and $\Psi_{1}$ and enable us to examine trapping effects quite readily.

The potential energy surfaces are approximated by Steele series with $\mathrm{NVG}=25$ [30] shells of reciprocal lattice vectors for the $\mathrm{NaCl}[\mathrm{KCl}]$ case and are constrained by a Lagrange multiplier construction ${ }^{6}$ to match the direct sum evaluations at the atop, center, and bridge sites of the unit cell for each perpendicular distance $z$ from the hydrogen monolayer. Contour plots of the He- $p(1 \times 1) \mathrm{H}_{2} / \mathrm{KCl}(001)$ potential energy in the $z=0$ plane (the plane of $\mathrm{H}_{2}$ centers) for $D Z L=1.2 \AA$ and $4.0 \AA$ are shown for one unit cell in Fig. 1. The two plots illustrate that the "hole" (area with a negative potential energy) at the center of the cell is larger for $D Z L=1.2 \AA$ than for $D Z L$ $=4.0 \AA$. This arises because the attractive part of the $\mathrm{He}-\mathrm{KCl}$ potential energy at $z=0$ is larger for $D Z L=1.2 \AA$ where the $\mathrm{KCl}$ surface is closer. However, the effects of the $z<0$ region are larger in the scattering calculations for $D Z L=4.0 \AA$ because the larger volume of this region outweighs the effect of the larger "hole" in the $z=0$ plane for $D Z L=1.2 \AA$.

In Paper III the closest approach of He to the $p(1 \times 1) \mathrm{H}_{2} /$ $\mathrm{NaCl}(001)$ monolayer was $z=0.4 \AA$ and there was a loss of up to $1.5 \%$ in the norm of $\Psi_{0}$ because the potential energy in part of the $z=0.4 \AA$ plane is less than the initial beam energy $E_{i}$. The potential energy at the unit cell center at $z=0$ is $1.8 \mathrm{meV}$ for the $\mathrm{NaCl}$ case and is in the range -5 to $-13 \mathrm{meV}$, depending on $D Z L$, for the $\mathrm{KCl}$ cases. The large potential energy at $z=-D Z L$ has the effect of reducing the loss of total norm (elastic + inelastic, $I_{e}+I_{i}$, Eq. (7)) to less than $0.1 \%$.

\section{Wave packet}

Equations (5) and (6) are solved with a wavepacket propagation scheme described in Ref. 5. Penetration of the wavepacket to the interlayer region now is included in all the calculations. The lateral motion is represented by plane waves, Eqs. (8) and (9), and the initial $z$-dependence of $\Psi_{0}$ is a Gaussian wavepacket. The values of the grid-size in the $z$-direction $\Delta z=0.06 \AA$ and integration time-step $\Delta t=0.04$ fs are as before. An absorbing potential at the large- $z$ end of the z-grid enables long-time calculations (now $120 \mathrm{ps}$ or more when there is transient trapping) that ensure that the scattering events go to completion and, with a determination of the $\mathrm{He}$
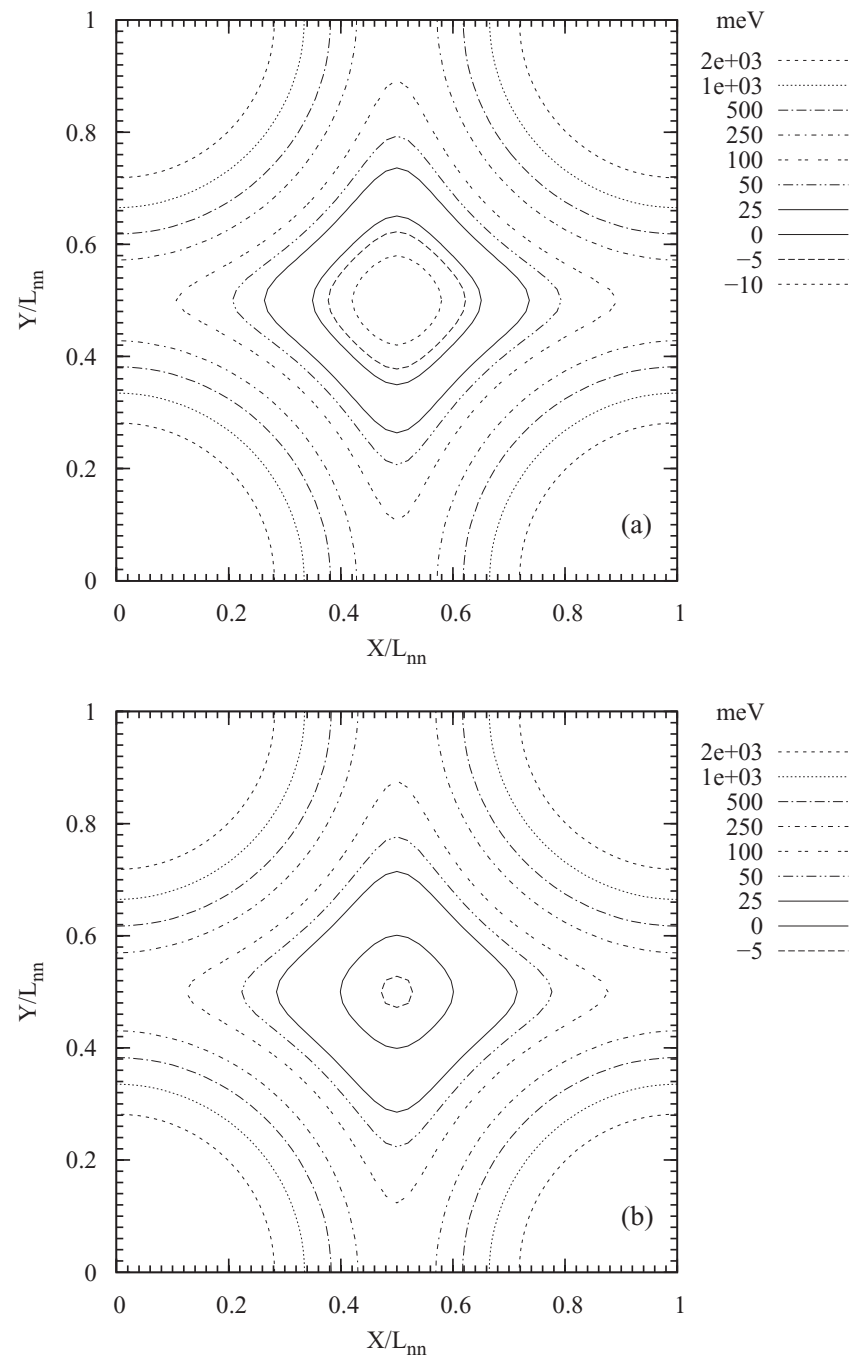

FIG. 1. 2D contour plots of the He- $p(1 \times 1) \mathrm{H}_{2} / \mathrm{KCl}(001)$ potential energy surface for a unit cell at $z=0$, the plane of the hydrogen molecules. The $\mathrm{H}_{2}$ are at the corners of this square cell with side $L_{n n}=4.44 \AA$. (a) The contours for $D Z L=1.2 \AA$ with a potential energy of $-13.3 \mathrm{meV}$ at the center. (b) The contours for $D Z L=4.0 \AA$ with a potential energy of $-5.4 \mathrm{meV}$ at the center.

flux into the absorbing potential, an accurate account of the intensity in all diffraction and inelastic scattering channels is maintained.

The calculations are done with two initial wavepacket widths to give a sense of the effect of the averaging over excitation cross sections in the experiment. The width $\sigma_{z}$ of the initial wavepacket is expressed in terms of the number $N W I$ of gridpoints as $\sigma_{z}=\Delta z N W I / \sqrt{2}$. The choices $N W I$ $=600$ and $N W I=100$ correspond to a somewhat finer or coarser energy resolution than in the $\mathrm{He} / \mathrm{H}_{2} / \mathrm{NaCl}(001)$ experiments. The increment in total energy that arises from the spread of the wavepacket is $\delta E=(\hbar / N W I \Delta z)^{2} / 4 m_{a}$ and is $2 \times 10^{-4} \mathrm{meV}$ for $N W I=600$.

\section{Scattering conditions}

The excitation spectra of shear horizontal (SH) and longitudinal acoustic (LA) phonons are taken to be the $\omega(\mathbf{Q})$ fit in Paper III to the experiments ${ }^{7}$ for $p(1 \times 1) \mathrm{H}_{2} / \mathrm{NaCl}(001)$. 
Quite different scattering geometries are treated for the $\mathrm{NaCl}$ and $\mathrm{KCl}$ cases.

The scattering for $p(1 \times 1) \mathrm{H}_{2} / \mathrm{NaCl}(001)$ is evaluated for scan curve conditions ${ }^{1}$ and a beam energy $E_{i}=26.5 \mathrm{meV}$ as in the experiments. The scan curve has the outgoing beam in the plane of incidence with a fixed angle $\theta_{S D}\left(=90.1^{\circ}\right.$ here $)$ between the incident and outgoing beam. In the calculations the plane of incidence is at an angle $\phi \neq 0^{\circ}$ relative to the $\Gamma \mathrm{M}$ $(\hat{y})$ axis of the monolayer and the phonon $\mathbf{Q}$ is in that plane. For the $\phi=0^{\circ}$ case and $\mathbf{Q}$ in the first Brillouin zone, there is no excitation of the $\mathrm{SH}$ mode. ${ }^{4} \mathrm{SH}-\mathrm{LA}$ pairs of scattering events at three angles of incidence $\theta_{i}$ and $\phi=1^{\circ}$ are treated in the present work. The diffraction conditions for a pair are the same, but the phonon excitations differ in order to satisfy the scan curve condition.

The scattering for $p(1 \times 1) \mathrm{H}_{2} / \mathrm{KCl}(001)$ is evaluated for $\phi=1^{\circ}$ and several $\theta_{i}$ for specific SH and LA phonons without the scan curve constraint.

\section{Approximating the Q-sum}

The full one-phonon approximation in Eqs. (5)-(7) allows for the excitation of phonons at all wavevectors $\mathbf{Q}$ in the first Brillouin zone and thus, effectively, includes a continuum of wavevectors. ${ }^{14}$ In the present work, we replace this continuum by single values of $\mathbf{Q}$ and polarization $\lambda$ so that there are only two coupled equations, Eqs. (5) and (6), to be solved. The strength of the coupling $\tilde{V}_{\mathbf{Q}, \lambda}$ depends on the number $N$ of atoms in the monolayer as $1 / \sqrt{N}$, Eq. (11) of Ref. 4. This implies that the norm of the wave function $\Psi_{1}$ for having excited a single specific phonon mode is $O(1 / N)$ and very small. In an experiment, however, there are finite resolutions $d \mathbf{Q}$ in $\mathbf{Q}$ and $d \Omega$ in the solid angle $\Omega$ for the final direction of the scattered atom, so the experimental intensity includes a factor $N|\mathbf{Q}| d|\mathbf{Q}| d \Omega$ and the $1 / \sqrt{N}$ dependence in $\tilde{V}_{\mathbf{Q}, \lambda}$ is compensated. Our approximation of a single $\mathbf{Q}$ and $\lambda$ and a specific value for $N$ is a rough way of allowing for the experimental resolution in wavevector and solid angle. Larger values of $N$ correspond to higher resolution and therefore to lower inelastic intensities.

We propagated the wavepacket with $N=10$, to get total inelastic norms $I_{i}$ of 0.1 to 0.2 , and made a few calculations with $N=5$ and $N=20$ to test how results varied with this choice. For the data presented in Table I, $I_{i}$ varies approximately as $1 / N$, although the feedback to $\Psi_{0}$ leads to departures from an exact proportionality to $1 / N$ at smaller $N$. Also, the conservation of total norm, Eq. (7), is maintained to within $0.1 \%$.

\section{Analysis}

We characterize the results of the calculations using the elastic and inelastic norms $I_{e} \equiv\left(\Psi_{0}, \Psi_{0}\right)$ and $I_{i} \equiv\left(\Psi_{1}, \Psi_{1}\right)$ and channel norms, e.g., $I_{i}(\mathbf{g}) \equiv\left(S_{\mathbf{Q}, \lambda}(\mathbf{g}), S_{\mathbf{Q}, \lambda}(\mathbf{g})\right)$,

$$
\left(S_{\mathbf{Q}, \lambda}(\mathbf{g}), S_{\mathbf{Q}, \lambda}(\mathbf{g})\right) \equiv \int_{-D Z L}^{\infty}\left|S_{\mathbf{Q}, \lambda}(\mathbf{g}, z, t)\right|^{2} d z .
$$

TABLE I. Effect of the value of $N$ on the self-consistent solution with coupling to only one phonon mode. Data for the $p(1 \times 1) \mathrm{H}_{2} / \mathrm{KCl}(001)$ model with $E_{i}=15.1 \mathrm{meV}, \theta_{i}=20^{\circ}, D Z L=1.2 \AA, N W I=600$, and a longitudinal acoustic phonon mode with $Q=0.718 \AA^{-1}$ and $\hbar \omega=8.47 \mathrm{meV} . I_{e}$ and $I_{i}$ are the total elastic and one-phonon inelastic norms; their sum should be 1 according to Eq. (7). The final column $N I_{i}$ would be independent of $N$ if $I_{i}$ scaled as $1 / N$ and this is nearly satisfied for $N=10$ and 20 .

\begin{tabular}{lcccc}
\hline \hline$N$ & $I_{e}$ & $I_{i}$ & $I_{e}+I_{i}$ & $N I_{i}$ \\
\hline 5 & 0.84741 & 0.15234 & 0.99975 & 0.7617 \\
10 & 0.89527 & 0.10447 & 0.99974 & 1.0447 \\
20 & 0.94047 & 0.05928 & 0.99975 & 1.1856 \\
\hline \hline
\end{tabular}

The partial norms $I_{e, i}$ (trap) of $\Psi_{0}$ and $\Psi_{1}$ in the range $-D Z L \leq z \leq 0$, defined by

$$
I_{e}(\operatorname{trap}) \equiv \int_{-\mathrm{DZL}}^{0} \sum_{\mathbf{g}}\left|\psi_{\mathbf{g}}(\mathrm{z}, \mathrm{t})\right|^{2} \mathrm{dz}
$$

with a similar definition for $I_{i}$ (trap), are used to track the size and time evolution of the trapping in the interlayer region, as in Fig. 2 which is discussed in Sec. III B. Factors that are
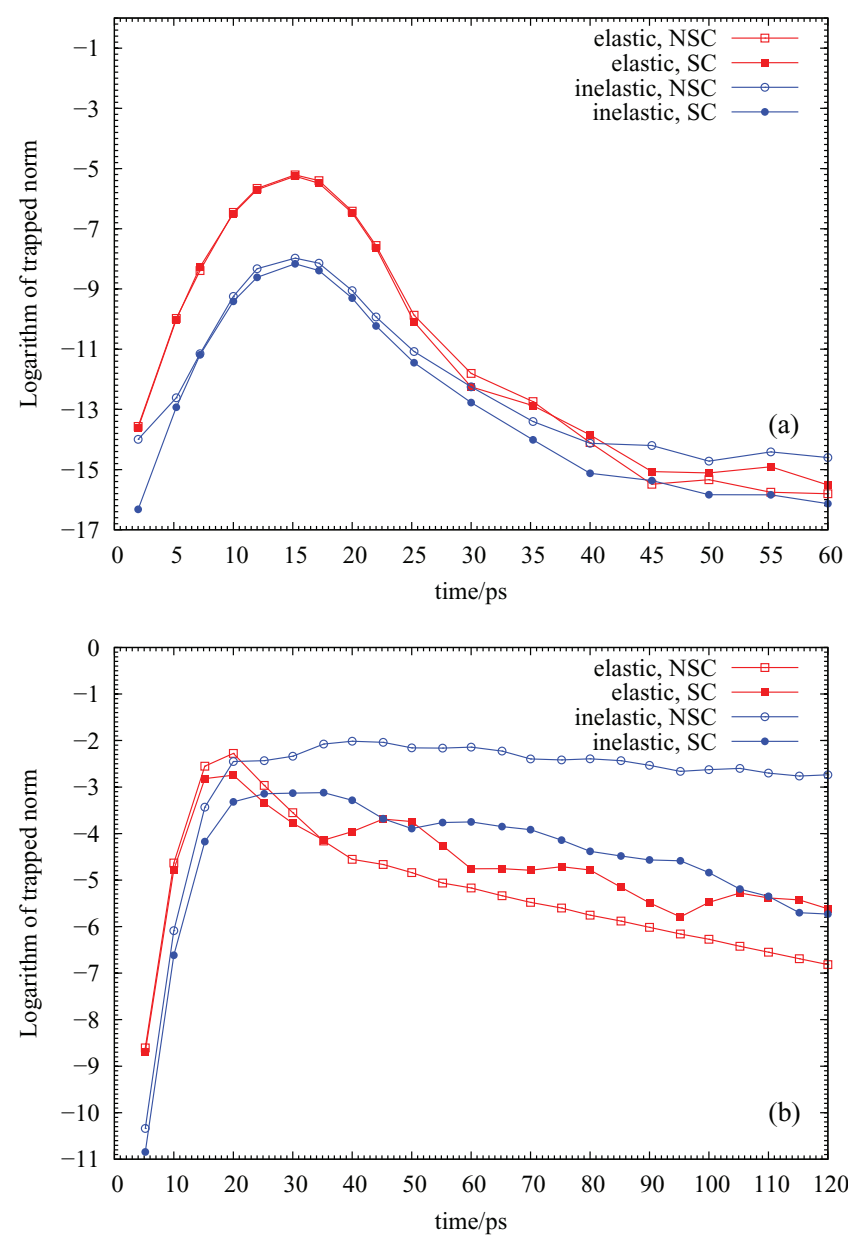

FIG. 2. The natural logarithms of the elastic and inelastic norms trapped between the planes of the $\mathrm{KCl}$ surface and the $\mathrm{H}_{2}$ monolayer, as a function of time for the SC and NSC calculations. The norms of $\Psi_{0}$ and $\Psi_{1}$ for $-D Z L$ $<z<0$ are evaluated for $\theta_{i}=20^{\circ}, \phi=1^{\circ}, E_{i}=15.1 \mathrm{meV}, N W I=600$ (nearly a monochromatic beam), and SH phonons with $(Q, \omega)=\left(0.718 \AA^{-1}\right.$, $6.61 \mathrm{meV}$ ). (a) $D Z L=1.2 \AA$. (b) $D Z L=4.0 \AA$. 
TABLE II. Total elastic and inelastic norms $I_{e}$ and $I_{i}$ at angle of incidence $\theta_{i}$ for scattering of $26.5 \mathrm{meV} \mathrm{He}$ atoms by the $p(1 \times 1) \mathrm{H}_{2} / \mathrm{NaCl}(001)$ monolayer as calculated ${ }^{6}$ in Paper III (NSC) and here with the self-consistent solution. $R_{i}$ is the ratio of the $I_{i}$ for LA and SH phonons, $I_{i}(\mathrm{LA}) / I_{i}(\mathrm{SH})$. The stated phonons ${ }^{\mathrm{a}}$ are those for which the inelastic scattering satisfies the scan curve condition with $\theta_{\mathrm{SD}}=90.1^{\circ}$. The azimuthal angle is $\phi=1^{\circ}$ relative to the $\Gamma \mathrm{M}$ axis of the monolayer, $N=10$, and $N W I=600$.

\begin{tabular}{llll}
\hline \hline$\theta_{i}$ & $46^{\circ}$ & $44.5^{\circ}$ & $37.2^{\circ}$ \\
\hline$I_{e}(\mathrm{LA})(\mathrm{SC})$ & 0.7407 & 0.7957 & 0.7111 \\
$I_{e}(\mathrm{SH})(\mathrm{SC})$ & 0.9056 & 0.8872 & 0.9022 \\
$I_{i}(\mathrm{SH})(\mathrm{SC})$ & 0.0941 & 0.1125 & 0.0975 \\
$R_{i}(\mathrm{SC})$ & 2.753 & 1.814 & 2.959 \\
$I_{i}(\mathrm{SH})(\mathrm{NSC})^{\mathrm{b}, \mathrm{c}}$ & 0.114 & 0.141 & 0.117 \\
$R_{i}(\mathrm{NSC})$ & 3.162 & 2.235 & 3.864 \\
$R_{i}(\mathrm{SC}) / R_{i}(\mathrm{NSC})$ & 0.871 & 0.810 & 0.766 \\
\hline \hline
\end{tabular}

${ }^{\mathrm{a}}(Q, \omega)$ in $\AA^{-1}$ and $\mathrm{meV}$ for the SH phonons at $\theta_{i}=46^{\circ}, 44.5^{\circ}$, and $37.2^{\circ}$ are $(0.865$, $6.87),(0.6,6.7)$, and $(-0.6,6.7)$ and for the LA phonons are $(1.02,8.27),(0.828,8.69)$, and $(-0.45,7.83)$.

${ }^{\mathrm{b}}$ By definition $I_{e}(\mathrm{NSC})=1$; in practice $I_{e}(\mathrm{NSC})$ departed from 1.00 by $0.5 \%$ at $46^{\circ}$ and $1.2 \%$ at $37.2^{\circ} . I_{e}+I_{i}$ for the $6 \mathrm{SC}$ calculations is maintained at 1.00 to within $0.04 \%$.

${ }^{c}$ Values from the work of Paper III, obtained with $N=1$, are restated here with $N=10$.

applied to the channel norms to generate final elastic and inelastic intensities ${ }^{6}$ in Paper III, i.e., a flux factor, the thermal average Debye-Waller factor, and an enhancement of phonon creation by scattering from thermally excited phonons, are not included in the present results. The SC approximation makes the ad hoc addition of that Debye-Waller factor at least partially redundant for this work. As before, ${ }^{5} \mathrm{~g}$-channels in $\Psi_{0}$ $\left[\Psi_{1}\right]$ are said to be open when the parallel kinetic energy in that channel is less than the final atom energy in $\Psi_{0}\left[\Psi_{1}\right]$.

Because of the ambiguity in the choice of $N$ for the single-Q approximation to the $\tilde{V}_{\mathbf{Q}, \lambda}$ coupling, we present many of the results as ratios of norms where the prefactor $N$ cancels. We use the ratio of the norms of the inelastic scattering for the LA and SH branches, $R_{i} \equiv I_{i}(\mathrm{LA}) / \mathrm{I}_{\mathrm{i}}(\mathrm{SH})$, and the ratio of the inelastic norm in the specular $(g=0)$ channel and the total inelastic norm in open channels, $I_{i, o}$, $F_{i} \equiv I_{i}(g=0) / I_{i, o}$. These enable us to follow trends in the SC and NSC calculations in parallel and $F_{i}$ is especially useful when the NSC calculation has a long-time loss in $I_{i}$ arising from norm trapped in closed channels. This arises in $D Z L=4.0 \AA \mathrm{NSC}$ cases for $p(1 \times 1) \mathrm{H}_{2} / \mathrm{KCl}(001)$ in
Sec. III B, where $I_{i, o} / I_{i}<0.7$ for times long enough that most of $I_{i}(\mathrm{SC})$ is in open channels, and we then use $I_{i, o}(\mathrm{NSC})$ for comparisons to the $\mathrm{SC}$ results.

\section{RESULTS}

\section{A. $p(1 \times 1) \mathrm{H}_{2} / \mathrm{NaCl}(001)$}

SC versions of the NSC calculations of Paper III were performed for pairs of inelastic scattering at energy $E_{i}=26.5 \mathrm{meV}$, three angles of incidence $\theta_{i}=46^{\circ}, 44.5^{\circ}$, and $37^{\circ}$ and azimuthal angle $\phi=1^{\circ}$. Table II shows the results of both versions of the calculations. The $(Q, \omega)$ of the SH and LA phonons that satisfy the scan curve condition at these $\theta_{i}$ are given in a footnote to Table II. The LA norm is much larger than the SH norm in each case, $R_{i}>1$, and the trends with $\theta_{i}$ for the NSC calculation follow the SC results. However, the feedback term in the $\mathrm{SC}$ calculation leads to smaller ratios $R_{i}$ and $R_{i}(\mathrm{SC}) / R_{i}$ (NSC) is in the range $0.77-0.87$. The stronger LA scattering has a greater reduction in the final elastic SC norm, as shown by the values for $I_{e}(\mathrm{LA})$ and $I_{e}(\mathrm{SH})$ in the first two lines of Table II. This comparison of SC and NSC results involves two changes in the calculation: (1) the inclusion of penetration of $\Psi_{0}$ and $\Psi_{1}$ to the $-D Z L<z<0$ region and (2) the implementation of the self-consistency by including the $\tilde{V}_{\mathbf{Q}, \lambda}$ term in Eq. (6) in the calculations. In a case where we treated the changes separately, the effect of the penetration was to change $I_{i}$ by only about $1 \%$ and it gave a small part of the total change.

The SC calculations thus confirm the conclusions of $\mathrm{Pa}-$ per III for the trends and relative magnitudes of the inelastic scattering strengths. Including the small penetration to the interlayer region between the monolayer and substrate leads to an improved conservation of the wave function norm without major changes in the rest of the results.

\section{B. $p(1 \times 1) \mathrm{H}_{2} / \mathrm{KCl}(001)$}

The calculations for the $p(1 \times 1) \mathrm{H}_{2} / \mathrm{KCl}(001)$ model are meant to explore what the consequences of a strong penetration to the interlayer region would be. We anticipated finding transient trapping of the elastic wave function $\Psi_{0}$ in the

TABLE III. Effect of the wavepacket width $N W I$ for the $p(1 \times 1) \mathrm{H}_{2} / \mathrm{KCl}(001)$ model with $E_{i}=15.1 \mathrm{meV}, N=10, D Z L=4.0 \AA$, and SH mode with $(Q, \hbar \omega)=\left(0.718 \AA^{-1}, 6.61 \mathrm{meV}\right)$. The total elastic and inelastic norms $I_{e}$ and $I_{i}$, their sum, and the fractions $F_{i}=I_{i}(g=0) / I_{i, o}$ are given. SC denotes results of the self-consistent calculations, Eqs. (5) and (6); NSC denotes results without the coupling term in Eq. (5). The SC-NSC pairs show a redistribution of strength caused by the mixing. The effect of changing NWI is much less in the SC calculation than in the NSC one.

\begin{tabular}{|c|c|c|c|c|c|c|}
\hline \multirow[b]{2}{*}{$\theta_{i}$} & \multicolumn{3}{|c|}{$N W I=600$} & \multicolumn{3}{|c|}{$N W I=100$} \\
\hline & $0^{\circ}$ & $15^{\circ}$ & $20^{\circ}$ & $0^{\circ}$ & $15^{\circ}$ & $20^{\circ}$ \\
\hline$I_{e}(\mathrm{SC})$ & 0.93406 & 0.94499 & 0.930475 & 0.92625 & 0.94223 & 0.93287 \\
\hline$I_{i}(\mathrm{SC})$ & $6.504 \times 10^{-2}$ & $5.340 \times 10^{-2}$ & $6.854 \times 10^{-2}$ & $7.301 \times 10^{-2}$ & $5.676 \times 10^{-2}$ & $6.619 \times 10^{-2}$ \\
\hline$I_{i}+I_{e}(\mathrm{SC})$ & 0.99910 & 0.99840 & 0.99902 & 0.99926 & 0.99899 & 0.99906 \\
\hline$F_{i}(\mathrm{SC})$ & $3.92 \times 10^{-3}$ & $1.34 \times 10^{-2}$ & $3.67 \times 10^{-2}$ & $3.34 \times 10^{-3}$ & $1.69 \times 10^{-2}$ & $2.95 \times 10^{-2}$ \\
\hline$I_{i, o}(\mathrm{NSC})^{\mathrm{a}}$ & $6.505 \times 10^{-2}$ & 0.220 & 0.111 & 0.118 & 0.186 & 0.287 \\
\hline$I_{i}(\mathrm{NSC})$ & $7.728 \times 10^{-2}$ & 0.426 & 0.165 & 0.181 & 0.288 & 0.514 \\
\hline$F_{i}(\mathrm{NSC})$ & $4.81 \times 10^{-3}$ & $4.22 \times 10^{-2}$ & $4.15 \times 10^{-2}$ & $5.41 \times 10^{-3}$ & $8.19 \times 10^{-2}$ & $5.60 \times 10^{-2}$ \\
\hline
\end{tabular}

${ }^{\mathrm{a}}$ These values are total inelastic norm in open channels. 
TABLE IV. Dependence of total inelastic scattering norm in open channels $I_{i, o}$ on interlayer spacing $D Z L$ for the $p(1 \times 1) \mathrm{H}_{2} / \mathrm{KCl}(001)$ model at $E_{i}=15.1 \mathrm{meV}, \theta_{i}=20^{\circ}, N=10$, and $N W I=600$. SC, NSC, and $F_{i}$ notation as in Table III; $R_{i, o}=I_{i, o}(\mathrm{LA}) / \mathrm{I}_{\mathrm{i}, \mathrm{o}}(\mathrm{SH})$. The phonon modes are the longitudinal acoustic and shear horizontal excitations at $Q=0.718 \AA^{-1}$ with energies 8.47 and $6.61 \mathrm{meV}$, respectively.

\begin{tabular}{|c|c|c|c|c|c|c|c|c|}
\hline \multirow[b]{2}{*}{$D Z L(\AA)$} & \multicolumn{4}{|c|}{$\mathrm{SC}$} & \multicolumn{4}{|c|}{ NSC } \\
\hline & $I_{i, o}(\mathrm{SH})$ & $R_{i, o}$ & $F_{i}(\mathrm{SH})$ & $F_{i}(\mathrm{LA})$ & $I_{i, o}(\mathrm{SH})$ & $R_{i, o}$ & $F_{i}(\mathrm{SH})$ & $F_{i}(\mathrm{LA})$ \\
\hline 1.2 & 0.0686 & 1.53 & 0.0133 & 0.0055 & 0.0978 & 1.49 & 0.0135 & 0.0188 \\
\hline 2.8 & 0.0800 & 2.25 & 0.0477 & 0.103 & 0.0894 & 2.40 & 0.0046 & 0.0967 \\
\hline 4.0 & 0.0679 & 1.81 & 0.0359 & 0.0755 & 0.111 & 5.88 & 0.0415 & 0.127 \\
\hline
\end{tabular}

interlayer region and that the time scale for the escape would be long. The first calculations were with the (NSC) wavepacket propagation used in Paper III and showed trapping of $\Psi_{0}$ that persisted $50-100$ ps for the $D Z L=2.8$ and 4.0 $\AA$ cases. However, the inelastic wave function $\Psi_{1}$ had a large component in the interlayer region that mostly did not escape to large $z$, as shown by the evolution of the open-channel norm $I_{i, o}$. At $\theta_{i}=15^{\circ}$ and $20^{\circ}$, much of the inelastic norm simply "disappeared" as time increased and the last term in Eq. (6) then acted more as a "sink" than a "source." This observation led us to the present self-consistent theory that includes the coupling term in the $\Psi_{0}$ propagation. The transient trapping involves a strong mixing of the $\Psi_{0}$ and $\Psi_{1}$ components of the total wave function because the coupling terms are large in the interlayer region. This results in a reduction of the inelastic norm and the mixing modifies the distribution over channels in $\Psi_{0}$.

The scattering for $p(1 \times 1) \mathrm{H}_{2} / \mathrm{KCl}(001)$ was evaluated for two examples of one-phonon creation events, a $\mathrm{SH}$ phonon of $(Q, \omega)=\left(0.718 \AA^{-1}, 6.61 \mathrm{meV}\right)$ and a LA phonon of $(Q, \omega)=\left(0.718 \AA^{-1}, 8.47 \mathrm{meV}\right)$. These are phonons from the spectrum ${ }^{7}$ of $p(1 \times 1) \mathrm{H}_{2} / \mathrm{NaCl}(001)$; the experimental data for the $\mathrm{SH}$ event at $E_{i}=15.1 \mathrm{meV}$ were shown in Paper III. Most of the calculations were for initial energy $E_{i}=15.1 \mathrm{meV}$ and trends were followed as a function of the angle of incidence $\theta_{i}$, effective interlayer spacing $D Z L$, and wavepacket width $N W I$.

Table III shows that the total inelastic norm in the SC solution, $I_{i}(\mathrm{SC})$, changes by about $10 \%$ as $N W I$ is increased at three angles $\theta_{i}$. This is very different from the
NSC results $I_{i}$ (NSC), where the changes with $N W I$ are much larger. [These comparisons are sensitive to whether the closed-channel contributions are included. The NSC ratios are $I_{i, o}(N W I=600) / I_{i, o}(N W I=100)\left\{I_{i}(N W I\right.$ $\left.=600) / I_{i}(N W I=100)\right\}=1.18\{1.48\}$ at $\theta_{i}=15^{\circ}$ and 0.33 $\{0.39\}$ at $\theta_{i}=20^{\circ}$.] The mixing in the interlayer region reduces the sensitivity to $N W I$ in the SC calculations. At long times, 80-100 ps after the turning-point time of the wavepacket [when the expectation value $\left(\Psi_{0}, z \Psi_{0}\right) /\left(\Psi_{0}, \Psi_{0}\right)$ is minimum], more than $90 \%$ of $I_{i}$ is in open channels for the SC solution but only $55 \%-65 \%$ is in open channels for the NSC one.

Examples from a series of calculations to explore the dependence on the interlayer width $D Z L$ are given in Table IV for $\theta_{i}=20^{\circ}$. The relative strength of the LA excitation to the SH excitation, $R_{i}$, is larger for larger values of $D Z L$ in the NSC calculations. This monotonic behavior is not found in the SC calculations, for which $R_{i}$ is largest at $D Z L=2.8 \AA$. We use $F_{i}$, the fraction of $I_{i, o}$ that is in the specular channel, to examine how the distribution over channels changes in the SC solution. A comparison of the SC and NSC columns for DZL $=2.8 \AA$ shows large changes in the ratios; the trends with $D Z L$ are quite uneven though. The feedback reduces the total inelastic norm; $I_{i}(\mathrm{SH})$ is smaller for SC than for NSC. There also is a large effect on the trapped norms, as shown in Fig. 2.

We made a few calculations at other incident energies to show the generality of the trapping and of the selfconsistent mixing of $\Psi_{0}$ and $\Psi_{1}$ in the interlayer region. Some results are given in Table V. For a low incident energy $E_{i}=10 \mathrm{meV}$, comparable to the energy $8.47 \mathrm{meV}$ of the

TABLE V. Inelastic scattering results at energies $E_{i}=10 \mathrm{meV}$ (LA excitation) and $E_{i}=26.5 \mathrm{meV}$ (SH excitation), phonon modes as in Table IV. $\mathrm{H}_{2} / \mathrm{KCl}(001)$ model for $\theta_{i}=20^{\circ}, N=10$, and $N W I=600$. Comparisons of SC and NSC calculations at two times $t$ long after the turning-point time (19.5 ps at 10 meV and $11.8 \mathrm{ps}$ at $26.5 \mathrm{meV}$ ).

\begin{tabular}{|c|c|c|c|c|c|c|c|c|}
\hline \multirow[b]{3}{*}{$t(\mathrm{ps})$} & \multicolumn{4}{|c|}{$E_{i}=10 \mathrm{meV}, \mathrm{LA}$} & \multicolumn{4}{|c|}{$E_{i}=26.5 \mathrm{meV}, \mathrm{SH}$} \\
\hline & \multicolumn{2}{|c|}{$\mathrm{SC}$} & \multicolumn{2}{|c|}{ NSC } & \multicolumn{2}{|c|}{$\mathrm{SC}$} & \multicolumn{2}{|c|}{ NSC } \\
\hline & 120 & 180 & 120 & 180 & 50 & 98 & 50 & 98 \\
\hline$I_{i}$ & $6.20 \times 10^{-2}$ & $6.15 \times 10^{-2}$ & $7.58 \times 10^{-2}$ & $7.77 \times 10^{-2}$ & 0.148 & 0.141 & 0.950 & 1.043 \\
\hline$I_{i}(\text { closed })^{\mathrm{a}}$ & $6.54 \times 10^{-4}$ & $1.06 \times 10^{-4}$ & $7.29 \times 10^{-3}$ & $8.84 \times 10^{-3}$ & $9.09 \times 10^{-3}$ & $1.26 \times 10^{-3}$ & $7.15 \times 10^{-2}$ & $7.54 \times 10^{-2}$ \\
\hline$I_{e}(\operatorname{trap})^{\mathrm{b}}$ & $7.24 \times 10^{-4}$ & $1.50 \times 10^{-4}$ & $1.97 \times 10^{-3}$ & $1.38 \times 10^{-3}$ & $5.84 \times 10^{-3}$ & $3.72 \times 10^{-4}$ & $4.05 \times 10^{-3}$ & $2.77 \times 10^{-4}$ \\
\hline$I_{i}(\operatorname{trap})^{\mathrm{b}}$ & $7.66 \times 10^{-4}$ & $1.10 \times 10^{-4}$ & $7.98 \times 10^{-3}$ & $9.17 \times 10^{-3}$ & $1.23 \times 10^{-2}$ & $8.11 \times 10^{-4}$ & 0.185 & $9.98 \times 10^{-2}$ \\
\hline$I_{i}(g=0)$ & $1.40 \times 10^{-2}$ & $1.41 \times 10^{-2}$ & $1.85 \times 10^{-2}$ & $1.88 \times 10^{-2}$ & $2.45 \times 10^{-3}$ & $2.46 \times 10^{-3}$ & $1.45 \times 10^{-2}$ & $1.50 \times 10^{-2}$ \\
\hline$F_{i}$ & 0.229 & 0.229 & 0.270 & 0.272 & $1.77 \times 10^{-2}$ & $1.76 \times 10^{-2}$ & $1.65 \times 10^{-2}$ & $1.55 \times 10^{-2}$ \\
\hline
\end{tabular}

${ }^{\mathrm{a}} I_{i}(\mathrm{closed})=I_{i}-I_{i, o}$ is the partial norm of $\Psi_{1}$ in closed channels, those with parallel kinetic energy greater than the final energy of the atom $E_{f}=E_{i}-\hbar \omega(Q)$.

${ }^{\text {b }}$ Partial norms of $\Psi_{0}$ and $\Psi_{1}$ in the range $-D Z L<z<0$, i.e., in the interlayer region. 
TABLE VI. Trends of the inelastic scattering for the $p(1 \times 1) \mathrm{H}_{2} / \mathrm{KCl}(001)$ model at $E_{i}=15.1 \mathrm{meV}$, with $N=10$ and $N W I=600$, except as noted. SC solutions for SH and LA excitations as in Table IV. The norm of total inelastic scattering in open channels $I_{i, o}$ and that in the specular channel $\mathbf{g}=0$ are given.

\begin{tabular}{|c|c|c|c|c|c|}
\hline \multirow[b]{2}{*}{$D Z L \AA$} & \multirow[b]{2}{*}{$\theta_{i}$} & \multicolumn{2}{|c|}{$\mathrm{SH}$} & \multicolumn{2}{|c|}{ LA } \\
\hline & & $I_{i, o}$ & $I_{i}(g=0)$ & $I_{i, o}$ & $I_{i}(g=0)$ \\
\hline \multirow[t]{2}{*}{1.2} & $0^{\circ}$ & 0.1328 & $4.14 \times 10^{-5}$ & 0.1317 & $1.02 \times 10^{-3}$ \\
\hline & $20^{\circ \mathrm{a}}$ & $6.86 \times 10^{-2}$ & $9.10 \times 10^{-4}$ & 0.1045 & $5.72 \times 10^{-4}$ \\
\hline \multirow[t]{2}{*}{2.8} & $0^{\circ \mathrm{b}}$ & $8.64 \times 10^{-2}$ & $3.50 \times 10^{-4}$ & $9.50 \times 10^{-2}$ & $1.48 \times 10^{-2}$ \\
\hline & $20^{\circ}$ & $8.00 \times 10^{-2}$ & $3.82 \times 10^{-3}$ & 0.180 & $1.86 \times 10^{-2}$ \\
\hline \multirow[t]{3}{*}{4.0} & $0^{\circ}$ & $6.41 \times 10^{-2}$ & $2.51 \times 10^{-4}$ & $8.94 \times 10^{-2}$ & $1.28 \times 10^{-2}$ \\
\hline & $15^{\circ}$ & $5.07 \times 10^{-2}$ & $6.79 \times 10^{-4}$ & $9.97 \times 10^{-2}$ & $5.45 \times 10^{-3}$ \\
\hline & $20^{\circ}$ & $6.79 \times 10^{-2}$ & $2.49 \times 10^{-3}$ & 0.1220 & $9.22 \times 10^{-3}$ \\
\hline
\end{tabular}

${ }^{\mathrm{a}}$ For the $\mathrm{H}_{2} / \mathrm{NaCl}(001)$ model at these scattering conditions the SH values are $4.72 \times 10^{-2}$ and $4.75 \times 10^{-4}$, respectively.

${ }^{\mathrm{b}} N W I=100$ for this case.

created LA phonon, a large fraction $\approx 0.25$ of $I_{i, o}$ is in the specular channel. The persistence of $I_{i}($ trap $)$ is much greater for the NSC calculation than for the SC one, as shown both by the amount in closed channels $I_{i}$ (closed) and by that specifically trapped in the interlayer region $I_{i}$ (trap), Fig. 3. The time dependence for $I_{i}$ (trap) of the LA phonon in Fig. 3 is similar to that of the SH phonon in Fig. 2(b). The persistence of $I_{i}$ (trap) is also much greater in the NSC calculation at $E_{i}=26.5 \mathrm{meV}$. At that energy, $I_{i}$ (NSC) is close to 1 , which shows a clear failure to maintain a reasonable account of the total norm.

In addition to determining the trapped norms of $\Psi_{0}$ and $\Psi_{1}$, we have analyzed the trapped probability density as a function of time and distance from the $\mathrm{H}_{2}$ monolayer. We evaluate $\left|\Psi_{0}\right|^{2}$ in several $z$-planes for $-D Z L<z<0$ and then calculate the average positions $\langle x\rangle$ and $\langle y\rangle$ and their variances $\sigma_{x}$ and $\sigma_{y}$ as a function of time in these planes. Figure 4 has examples of contour plots of the $\left|\Psi_{0}\right|^{2}$ in the planes $z=-1.0$ $\AA$ and $z=-2.44 \AA$, evaluated 20 ps after the turning-point time of the wavepacket in an SC calculation with $E_{i}=15.1$ $\mathrm{meV}, \theta_{i}=15^{\circ}, N W I=600, D Z L=4.0 \AA$, and creation of SH phonons with $(Q, \omega)=\left(0.718 \AA^{-1}, 6.61 \mathrm{meV}\right)$. The con-

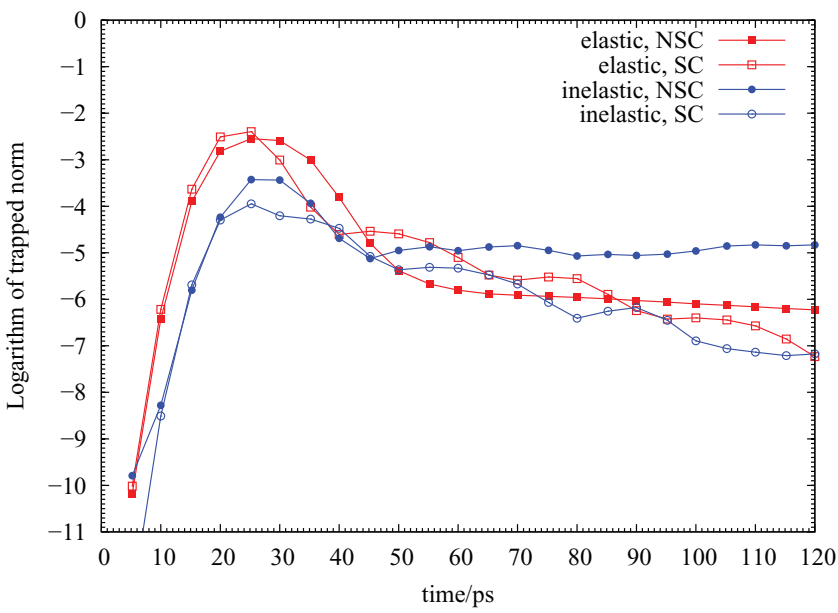

FIG. 3. The natural logarithms of the trapped elastic and inelastic norms, defined as in Fig. 2, as a function of time for the SC and NSC calculations. The scattering event has $\theta_{i}=20^{\circ}, \phi=1^{\circ}, E_{i}=10.0 \mathrm{meV}, N W I=600$, $D Z L=4.0 \AA$, and LA phonons with $(Q, \omega)=\left(0.718 \AA^{-1}, 8.47 \mathrm{meV}\right)$. tour plots largely reflect the symmetry of the square cell. The lateral extent of $\left|\Psi_{0}\right|^{2}$ near the $\mathrm{H}_{2}$ layer, Fig. 4(a) $(z=-1.0)$, is smaller than further away, Fig. 4(b) $(z=-2.44)$, due to the short-range repulsive interaction of $\mathrm{He}$ with $\mathrm{H}_{2}$ at the unit cell corners. Figure 5 shows the average positions $\langle x\rangle,\langle y\rangle$ and their variances $\sigma_{x}$ and $\sigma_{y}$ in the plane $z=-1.90 \AA$ as a function of time. The large variances demonstrate that the probability density is spread widely over the plane; if the distributions were uniform, the variance would be $L_{n n} / \sqrt{12}$ ( $=1.28 \AA$ for
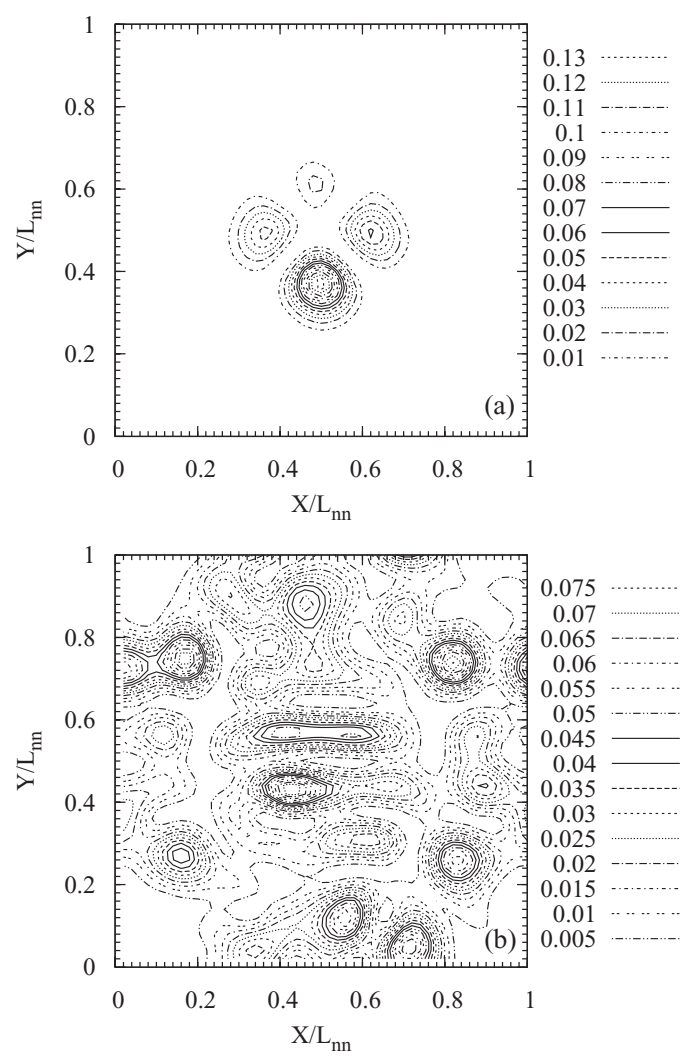

FIG. 4. 2D contour plots of $\left|\Psi_{0}\right|^{2}$ in the interlayer region from an SC calculation for a scattering event with $E_{i}=15.1 \mathrm{meV}, \theta_{i}=15^{\circ}, N W I=600$, $D Z L=4.0 \AA$, and SH phonons with $(Q, \omega)=\left(0.718 \AA^{-1}, 6.61 \mathrm{meV}\right)$. Evaluated in two planes at $20 \mathrm{ps}$ after the turning point time of the wavepacket, with trapped elastic norm about $50 \%$ of its maximum value. (a) $z=-1.0 \AA$. (b) $z=-2.44 \AA$. 


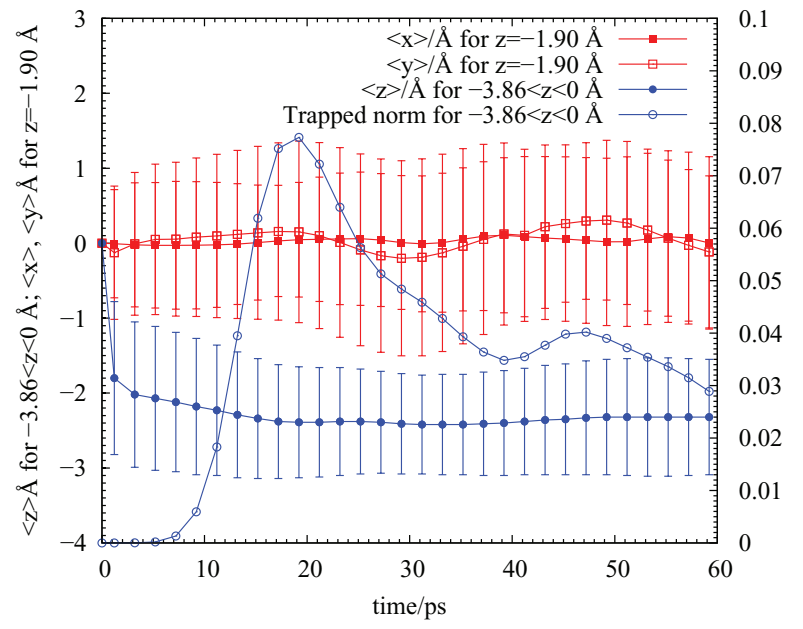

FIG. 5. The average positions in the $x$ and $y$ directions, $\langle x\rangle,\langle y\rangle$, and their variances (error bars) as function of time in the plane $z=-1.90 \AA$, evaluated with $\left|\Psi_{0}\right|^{2}$ of the same scattering event as in Fig. 4. Also shown are the average position in the $z$ direction, $\langle z\rangle$, calculated for $\left|\Psi_{0}\right|^{2}$ on the interval $-D Z L<z<0$ and the trapped elastic norm (right ordinate axis) to set the time relative to the evolution of the scattering event. The turning-point time is at $15.32 \mathrm{ps}$ and the trapped elastic (inelastic) norm is maximum at 18.8 (19.6) ps.

the $\mathrm{KCl}$ case) and this is the scale shown in the figure. Also shown are the average $\langle z\rangle$ and trapped elastic norm for $-D Z L$ $<z<0$ to show the relation of the time-dependence of the other averages to the evolution of the scattering event. The averages and their variances have little time dependence, although the total trapped norm decreases from 0.08 at its maximum to 0.03 at the largest time in the figure. A rather uniform distribution is established early in the scattering event and is maintained even when $I_{e}$ (trap) has decayed to $1 / 3$ of its maximum value. After a transient period, $\langle z\rangle$ is practically timeindependent. We interpret these data as showing that multiple scattering of the $\mathrm{He}$ atom between the substrate and the hydrogen layer effectively establishes a stationary distribution with a normalization that follows $I_{e}$ (trap). However, the variances $\sigma_{x}$ and $\sigma_{y}$ depend on $D Z L$ and are smaller for $D Z L$ $=1.2$ and $2.8 \AA$ (not shown) than for the $4.0 \AA$ case shown here, which is consistent with the distributions in Fig. 4.

Our original goal was to examine the effects of penetration of the He atom to the interlayer region. In Table VI we give our best estimate for trends of the inelastic scattering with $D Z L$ and $\theta_{i}$. The relative strength of total LA inelastic scattering to that of the $\mathrm{SH}, R_{i}$, increases with increasing thickness of the interlayer region mostly because of an increase in the absolute strength for the LA.

\section{CONCLUSIONS}

We conclude that the effects of interlayer trapping are seriously overestimated without the self-consistent solution. This is another manifestation of the strong-coupling scattering of thermal energy He atoms. The portion of the wave function that penetrates to the interlayer region experiences strong elastic and inelastic scattering. However, the trapping keeps the inelastic wave there long enough that the coupling to the phonons restores much of the lost energy and trans- forms much of this part of the inelastic wave back to the elastic component. The final inelastic strength remains large, but is not as large as estimated without allowing for the feedback, and the distribution over elastic channels is much changed by the penetration and trapping.

The self-consistent solutions reported here are based on a serious simplification of the formalism, to only one phonon excitation (one $\mathbf{Q}$ and polarization). To do calculations with a sampling of 25 or so points in the Brillouin zone will require considerably more computational resources than we now have, but this extension does seem feasible in the light of the expanding capabilities of parallel computation for such a problem and work is in progress.

The problem of combining diffractive and inelastic scattering in an internally consistent theory is of long standing and is particularly difficult when both types of scattering are strong. This is the situation here, with a target that is highly corrugated to the extent that the He atoms may penetrate it. The energy ranges we treat are intermediate between very low energies, where one-phonon losses would suffice to trap the incoming atom, ${ }^{20}$ and higher energies, where multiphonon processes dominate the inelastic scattering. Perhaps surprisingly, we find that the self-consistent formulation of the onephonon inelastic scattering moderates the sensitivity of the calculations to details such as the width of the wave packet. The next stages in the development of the theory should give new insights into the scattering effects that are summarized in empirical Debye-Waller factors.

\section{APPENDIX: GEOMETRICAL OPTICS APPROXIMATION}

As a guide to interpreting the penetration of the elastic wave function $\Psi_{0}$ to the space between the monolayer and the substrate, we construct a geometrical optics approximation for the propagation of rays incident on the potential energy surface from above (positive $z$ ). Determining which rays are able to pass through the "hole" in the $z=0$ plane, allowing for multiple reflections, gives the transmitting fraction of the unit cell as a function of incident angle $\theta_{i}$. This fraction decreases monotonically as $\theta_{i}$ increases. The trapped norm $I_{e}$ (trap) in the wavepacket calculation has more complex dependence on $\theta_{i}$. However its maximum has a large decrease from $0^{\circ}$ to $43^{\circ}$ and $75^{\circ}$, in qualitative agreement with the ray tracing results. This is remarkable because the calculation of the transmitted rays does not include such effects as the multiple scattering of rays in the interlayer region, details of the potential energy surface there, and the role of attractive forces on the atom at $z>0$.

The ray tracing is done in a mid-plane at $y=L_{n n} / 2$ of the square unit cell illustrated in Fig. 1. The $x-z$ surface of potential energy equal to the incident atom energy $E_{i}$ is constructed, using $E_{i}=15.1 \mathrm{meV}$ and $D Z L=1.2$ and $4.0 \AA$ for $\mathrm{H}_{2} / \mathrm{KCl}(001)$ and $26.5 \mathrm{meV}$ and $D Z L=1.2 \AA$ for $\mathrm{H}_{2} / \mathrm{NaCl}(001)$. Then rays incident at angle $\theta_{i}$ relative to $\hat{z}$ at a specified position $x$ are traced through successive geometric reflections (angle of incidence at the surface equal to angle of reflection) to determine whether they are transmitted "down" through the $z=0$ plane or are reflected "up" away from the surface. Up to 8 successive reflections at the surface 

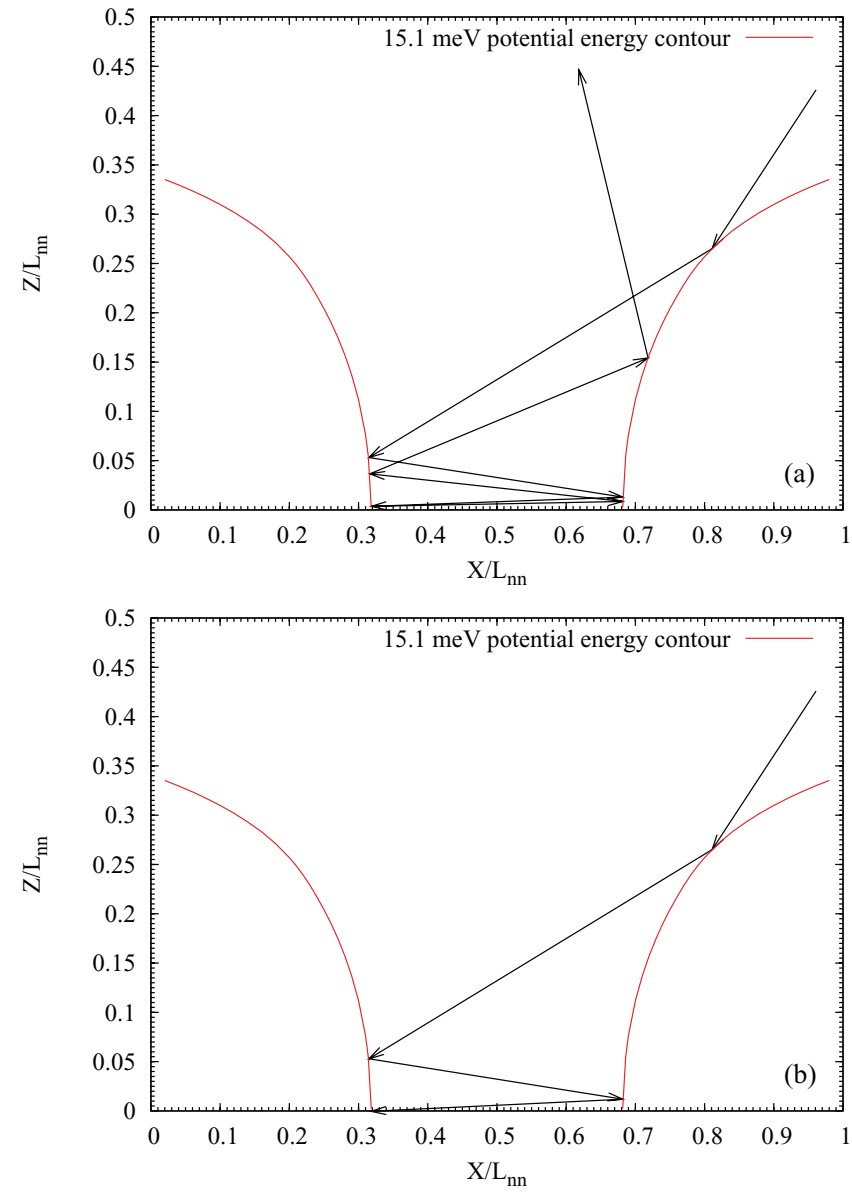

FIG. 6. Examples of ray tracing in which the incident ray is reflected without reaching $z=0$ and in which it reaches the $z=0$ plane after multiple reflections ("transmitted"). Angle of incidence $\theta_{i}=43^{\circ}$ and first contact of ray with reflecting surface is $x_{0} / L_{n n}$. The arrows denote the direction of the ray. $\mathrm{H}_{2} / \mathrm{KCl}(001)$ model with $L_{n n}=4.44 \AA, D Z L=4.0 \AA$, and $E_{i}=15.1 \mathrm{meV}$. (a) Reflection, $x_{0} / L_{n n}=0.8110$. (b) Transmission, $x_{0} / L_{n n}=0.81094$. The edge $x_{e}$ of the transmitting strip is at $x_{e} / L_{n n} \simeq 0.811$.

are evaluated for a given incident ray and this is enough to assign the ray as transmitted or reflected and determine the edges $x_{e}$ of the transmitting strip to $\pm 0.001 L_{n n}$. Two examples are shown in Fig. 6. For the angle of incidence shown there, $\theta=43^{\circ}$, the edge of the transmitting strip is at $x_{e} / L_{n n}$ $\simeq 0.811$. Rays with impact position farther from the "hole" are reflected while those closer to the "hole" are transmitted. We present the result for the transmitting width $\Delta x_{e}(\theta)$ in Fig. 7 in the form of the ratio $w_{r}=\Delta x_{e}(\theta) / \Delta x_{e}(0)$.

As a measure of the amount of wavepacket transmitted in the scattering calculations, we follow the trapped elastic norm as a function of time and use the maximum value. This depends on the potential energy surface at $z<0$, the inelastic scattering (SH or LA), and the width $N W I$ as well as on $\theta$, so we compare trends within one series set by $D Z L, N W I$, and the phonon. Results for the ratio $w_{w}$ between the maximum trapped elastic norm at $\theta_{i}$ and the maximum trapped norm at $\theta=0^{\circ}$ are plotted in Fig. 7. There are two $\mathrm{KCl}$ series and one $\mathrm{NaCl}$ series of angles $\theta_{i}$. There is qualitative agreement between the trends for $w_{r}$ and $w_{w}$ in the three series. The ray tracing ratios decrease monotonically to very small values as $\theta_{i}$ increases while the wavepacket ratios have an intermediate maximum at $15^{\circ}-20^{\circ}$ for the $\mathrm{KCl}$ series and then decrease
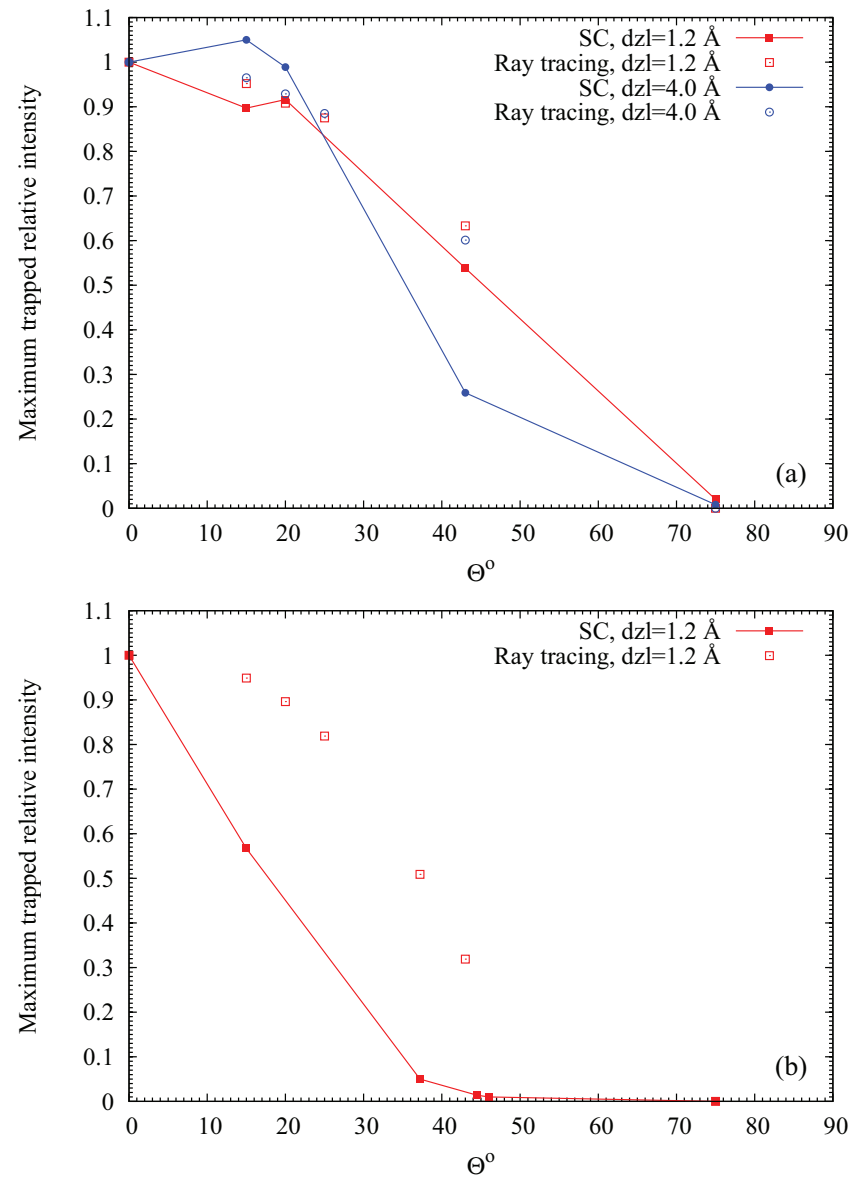

FIG. 7. The ratio $w_{w}$ of the maximum trapped elastic norm for wavepacket incident at $\theta$ to that at $\theta=0^{\circ}$ and the ratio $w_{r}$ of the ray-tracing transmitted fraction at $\theta$ to that at $\theta=0^{\circ}$. SC calculations for the specified event. (a) $\mathrm{KCl}, E_{i}=15.1 \mathrm{meV}, N W I=600, \phi=1^{\circ}$, and SH phonon $\left(0.718 \AA^{-1}, 6.61\right.$ meV). (b) $\mathrm{NaCl}, E_{i}=26.5 \mathrm{meV}, N W I=600 \phi=1^{\circ}$, and SH phonons as in Table II for $37.2^{\circ}, 44.5^{\circ}$, and $46^{\circ}$. For the other angles, SH phonon with $\left(0.685 \AA^{-1}, 6.87 \mathrm{meV}\right)$. For $\theta=75^{\circ}$ all ray tracing results gave zero which is difficult to see in the graph because the points are on top of each other.

more sharply than in the ray-tracing. We note that the ray tracing results in most cases are larger than the wavepacket results probably because the ray tracing does not include effects such as impedance mismatching that arise in wave propagation. There are large quantitative differences with the ratios obtained from the NSC calculations $(N W I=100, \mathrm{KCl}(001)$ cases) where maxima of 1.3-1.7 are found; i.e., the NSC approximation is misleading also for this aspect of the trapping.

${ }^{1}$ A. P. Graham, Surf. Sci. Rep. 49, 115 (2003).

${ }^{2}$ L. W. Bruch, R. D. Diehl, and J. A. Venables, Rev. Mod. Phys. 79, 1381 (2007), and references contained therein.

${ }^{3}$ B. Gumhalter, Phys. Rep. 351, 1 (2001); J. Phys.: Condens. Matter 24, 104015 (2012).

${ }^{4}$ L. W. Bruch and F. Y. Hansen, J. Chem. Phys. 122, 114714 (2005). (Denoted as Paper I.)

${ }^{5}$ F. Y. Hansen and L. W. Bruch, J. Chem. Phys. 127, 204708 (2007). (Denoted as Paper II.)

${ }^{6}$ L. W. Bruch, F. Y. Hansen, and F. Traeger, J. Chem. Phys. 134, 194308 (2011). (Denoted as Paper III in the text.) The channel norms denoted by $N$ with subscripts in Papers I-III are denoted by $I_{\ldots}$ here.

${ }^{7}$ F. Traeger, Ph.D. dissertation, Max-Planck-Institut für Strömungsforschung and Universität Göttingen, Göttingen, Germany, 2001; F. Traeger and J. P. Toennies, J. Phys. Chem. B 108, 14710-14725 (2004); J. P. Toennies and F. Traeger, J. Phys.: Condens. Matter 19, 305009 (2007). 
${ }^{8}$ F.-M. Tao, J. Chem. Phys. 100, 4947-4954 (1994).

${ }^{9}$ D. Spelsberg and W. Meyer, J. Phys. Chem. 100, 14637-14642 (1996).

${ }^{10}$ M. C. Vargas and W. L. Mochán, Surf. Sci. 406, L619-L620 (1998); 409, 130-136 (1998).

${ }^{11}$ R. D. Diehl, T. Seyller, M. Caragiu, G. S. Leatherman, N. Ferralis, K. Pussi, P. Kaukasoina, and M. Lindroos, J. Phys.: Condens. Matter 16, S2839S2862 (2004).

${ }^{12}$ B. H. Choi and R. T. Poe, J. Chem. Phys. 83, 1330 (1985).

${ }^{13}$ R. Manson and V. Celli, Surf. Sci. 24, 495 (1971).

${ }^{14}$ A. Šiber and B. Gumhalter, Phys. Rev. B 71, 081401 (2005).
${ }^{15}$ D. Eichenauer and J. P. Toennies, J. Chem. Phys. 85, 532 (1986).

${ }^{16}$ A. Šiber and B. Gumhalter, J. Phys.: Condens. Matter 20, 224002 (2008).

${ }^{17}$ S. Daon, E. Pollak, and S. Miret-Artés, J. Chem. Phys. 137, 201103 (2012).

${ }^{18}$ J. Heidberg, A. Voßberg, M. Hustedt, M. Thomas, S. Briquez, S. Picaud, and C. Girardet, J. Chem. Phys. 110, 2566-2578 (1999).

${ }^{19}$ L. W. Bruch, M. W. Cole, and E. Zaremba, Physical Adsorption: Forces and Phenomena (Dover, Mineola, NY, 2007).

${ }^{20}$ A. Šiber, B. Gumhalter, and C. Wöll, J. Phys.: Condens. Matter 14, 5913 (2002). 